\title{
Rubens y Van Dyck: Tres nuevas pinturas de Victor Wolfvoet bajo su estela
}

\author{
Three new Paintings by Victor Wolfvoet in the Footsteps of \\ Rubens and Van Dyck
}

\begin{abstract}
Matías Díaz Padrón ${ }^{1}$
Académie Royale d’Archéologie de Belgique Instituto Moll. Centro de investigación en pintura flamenca
\end{abstract}

Resumen: Se añaden al catálogo de Victor Wolfvoet tres nuevas pinturas con anteriores atribuciones confusas al taller de Rubens, y a otros pintores como Peter Thys y Willem van Herp. Este estudio contribuye así a definir la personalidad de Victor Wolfvoet, pintor estrechamente vinculado a Rubens y Van Dyck, cuyas composiciones repite en pequeño formato y con un estilo bien definido.

Palabras clave: Victor Wofvoet; Peter Thys; Willem van Herp; Simon de Vos; pintura flamenca; siglo XVII, Calvario; Martirio de San Sebastián; Santa Cecilia.

Abstract: This article adds three new paintings to Victor Wolfvoet's catalogue. These paintings were previously confusedly attributed to the workshop of Rubens and to other painters such as Peter Thys and Willem van Herp. This study contributes to defining the personality of this painter closely linked to Peter Paul Rubens y Anton van Dyck, whose compositions he reproduced in small format and with a well-defined style.

Keywords: Victor Wofvoet; Peter Thys; Willem van Herp; Simon de Vos; Flemish painting; $17^{\text {th }}$ century; Calvary; Martyrdom of Saint Sebastian; Saint Cecilia.

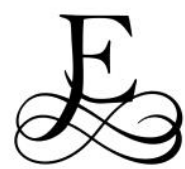

s objeto de este artículo dar a conocer tres nuevas pinturas que por motivos artísticos y técnicos restituimos a Victor Wolfvoet (Amberes, 1612-1652), pintor flamenco no muy conocido pero

1 () https://orcid.org/0000-0002-5137-7583

(C) 2021 Philostrato. Revista de Historia y Arte 
recuperado en los últimos años gracias a diversos artículos que van poniendo las bases de la producción y estilo de este artista que trabajó en la estela de Peter Paul Rubens y Anton van Dyck². Sorprende esta estrecha vinculación con los modelos de ambos, a los que copia con servilismo extremo.

Victor Wolvoet nace en Amberes el 4 de mayo de 1612. Formado en el taller de su padre, así se recoge en la guilda de pintores de la ciudad en 1644 y $1645^{3}$, año en que registra a Jan-Franssoes Olimaers como discípulo en su taller ${ }^{4}$. Martin y Schepers explican que tras su formación previa pudo estar trabajando un tiempo en el taller de Rubens, de ahí que su presencia como artista independiente en la guilda de pintores sea de fecha tardía, ocho años antes de su fallecimiento en $1652^{5}$. Combinó sus tareas artísticas con el mercado, colaborando activamente con Mathias Musson ${ }^{6}$. Tras su fallecimiento el 23 de octubre de 1652, se levanta inventario de los objetos dejados en su casa familia, en la calle Sint Jans. Se recogen unos 585 objetos artísticos entre pinturas y grabados de los más diversos autores, destacando copias siguiendo a Rubens, Van Dyck y Gerard Seghers ${ }^{7}$. Es enterrado en la iglesia de San Jorge de la ciudad del Escalda, al lado del altar con un retablo con las Obras de Misericordia ${ }^{8}$.

La estrecha relación que parece que tuvo con uno de los marchantes de obras de arte más importantes de mediados del siglo XVII como fue Mathias Musson, explica la presencia tan habitual de su producción en tierras hispánicas. Sobre todo, copias de maestros, que realiza tanto sobre tabla co-

2 Matías Díaz Padrón, "Dos cobres de Víctor Wolfvoet en el Museo San Carlos de México", Boletín del Seminario de Estudios de Arte y Arqueología, no 65, (1999), pp. 323-328; M. Díaz Padrón, "Tres cobres restituidos a Víctor Wolfvoet, el más fiel seguidor de Rubens", Archivo Español de Arte, no 316, (2006), pp. 403-425; M. Díaz Padrón, "Una adoración de los reyes de Víctor Wolfvoet en el coleccionismo madrileño", Tendencias del Mercado del Arte, no 18, (2008), pp. 32-33; M. Díaz Padrón, "Tres nuevos cobres de Víctor Wolfvoet con la Paz y la Guerra bajo la consigna de Rubens", Archivo Español de Arte, 337, LXXXV, (2012), pp. 88-94; M. Díaz Padrón, "Una Andrómeda con Perseo y Pegaso en la lejanía de Víctor Volfvoet atribuida a David Teniers en la galería Christie's de Nueva York", Tendencias del Mercado del Arte, no 53, (2012), pp. 92-94; M. Díaz Padrón, "Un Calvario de Víctor Wolfvoet en la colección Gerstenmaier", Tendencias del Mercado del Arte, no 68, (2014), pp. 82-84; Gregory Martin y Bert Schepers, "Two Antwerp cabinets decorated by Victor Wolfvoet II", The Burlington Magazine, 158, no 1363, (2016), pp. 793-802; M. Díaz Padrón, "La Visitación de Saint- Jacques de Amberes ¿ Victor Wolfvoet o Simon de Vos?", Philostrato. Revista de Historia y Arte, no 1 (2017), pp. 43-55 (DOI: https://doi.org/10.25293/philostrato.2017.03); M. Díaz Padrón, "Algo más sobre Victor Wolfvoet. Una segunda réplica de la Crucifixión atribuida a Abraham van Diepenbeeck", Philostrato. Revista de historia y arte, no extraordinario 1, (2018), pp. 296-304 (DOI: https://doi.org/10.25293/philostrato.2018.11); M. Díaz Padrón, "Una segunda réplica de la Crucifixión de Victor Wolfvoet atribuida a Abraham Diepenbeeck", Tendencias del mercado del arte, no 136, (2020), pp. 74-75; Bert Schepers, "Copy/paste Rubens: the art of Victor II Wolvoet. Newly identified works in the Spanish Royal Collections", Las copias de obras maestras de la pintura en las colecciones de los Austrias y el Museo del Prado. Actas del congreso internacional. Museo del Prado, junio, 2017, (Madrid: Museo Nacional del Prado, 2021), pp. 106-115.

3 Philips Rombouts y Theodor van Lerius, De Liggeren en Andere Historische Archieven, vol. 2, (s'Granvenhage, 1872/1961), pp. 161-162.

${ }^{4}$ Rombouts y Van Lerius, De Liggeren, p. 163.

${ }^{5}$ Martin y Schepers, "Two Antwerp cabinets", p. 793; Schepers, "Copy/paste Rubens", p. 107.

${ }^{6}$ Así parece ser ante la anotación del inventario postmorten, en la que se explica que el señor Mathías Musson se personó en la casa del difunto reclamando las pinturas por las que había pagado un salario de 270 gulden a su socio, Victor Wolfvoet. E. Duverger, Antwerpse Kunstinventarissen uit de Zeventiende Eeuw, vol. 6, Fontes Historiae Artis Neerlandicae, (Brussel: Paleis der Academiën, 1992), p. 361.

7 Duverger, Antwerpse Kunstinventarissen, vol. 6, pp. 344, 347, 352, 353, 354, 355, 356, 360.

${ }^{8}$ Inscriptions funéraires et monumentales de la province de Anvers, vol. 2., Eglisés paroissiales, part. 1, (Anvers: Buschmann, 1863), p. 491. 


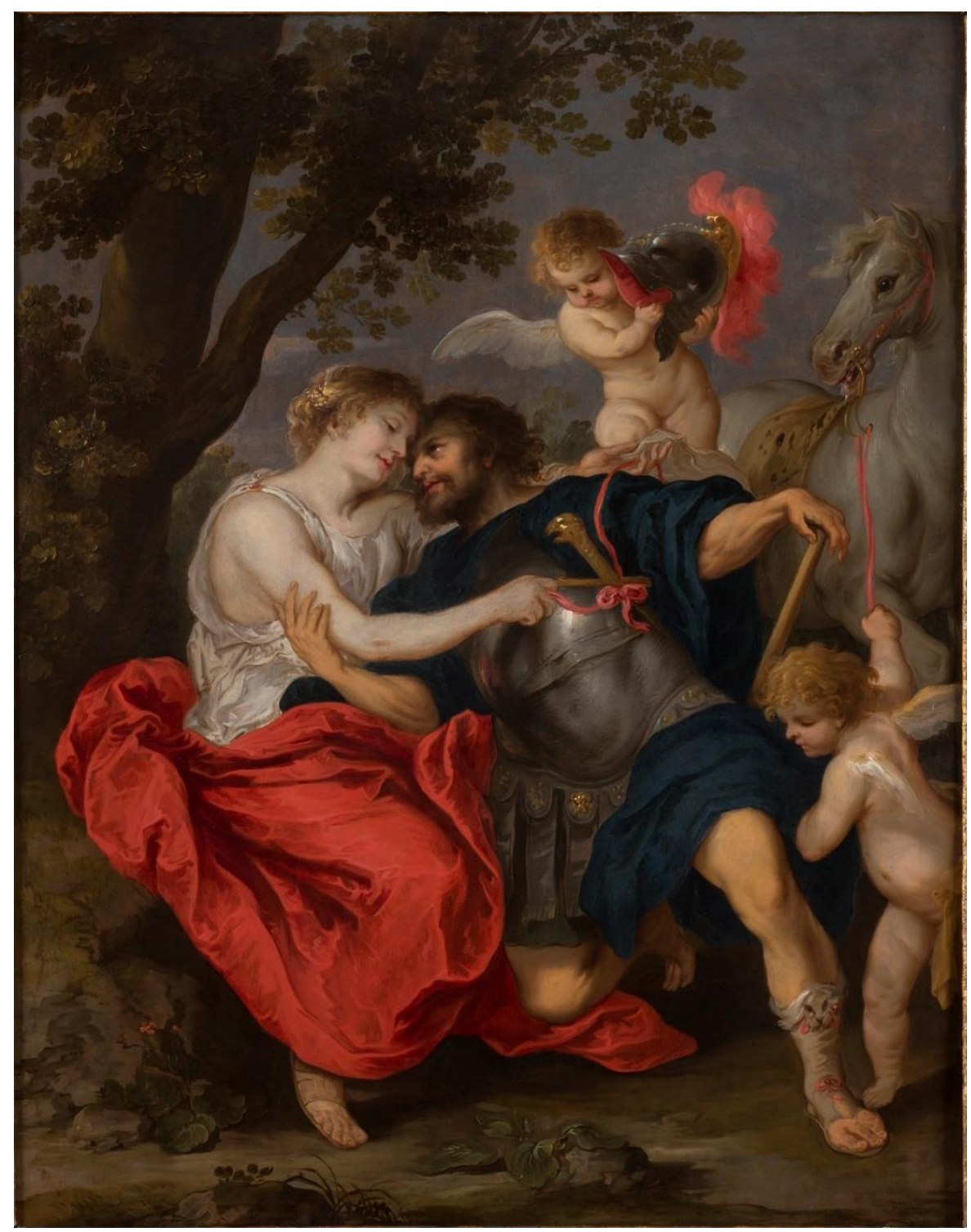

Fig. 1. Victor Wolfvoet II, Venus desarmando a Marte, ca. 1640. Valencia, @ colección Epiarte (inv. 894)

mo sobre cobre ${ }^{9}$, con bellos ejemplos en colecciones públicas y privadas españolas ${ }^{10}$. (Fig. 1)

Es el caso del Calvario sobre cobre de colección privada $(33,3 \times 26$ cm.),

\begin{abstract}
'Sobre la dependencia de modelos de Rubens y su copia directa en el taller o a través de copias, dibujos y grabados siguiendo las composiciones del maestro, véase Martin y Schepers, "Two Antwerp cabinets", pp. 799 y 802 ; Schepers, "Copy/paste Rubens", pp. 107-108 y 110. Agradezco a Ana Diéguez Rodríguez esta información.

10 Díaz Padrón, "Tres cobres restituidos", pp. 404-407; David García Cueto, "The Reflection of `Flandes 'in the Spanish Royal Collections after Rubens in the Patrimonio Nacional" en Copies of Flemish Masters in the Hispanic World (1500-1700). Flandes by Substitution, ed. Eduardo Lamas y David García Cueto, (Turnhout: Brepols, 2021), p. 199; Schepers, "Copy/paste Rubens", pp. 109-112. Este último incluye, además de los cobres de Patrimonio Nacional (inv. n.o 10003190 y n.0 10003191) la serie de cobres de la sacristía mayor de la catedral del Salvador de Zaragoza, y dos de la Sagrada Familia en el museo diocesano de Barbastro-Monzón. Todos ellos reproducidos en M. Carmen Lacarra Ducay y Juan C. Lozano López, coords., Aragón y Flandes. Un encuentro artístico. Siglos XV-XVII, (Zaragoza: Universidad, 2015), pp.
\end{abstract} 115,117 y $212-218$. 


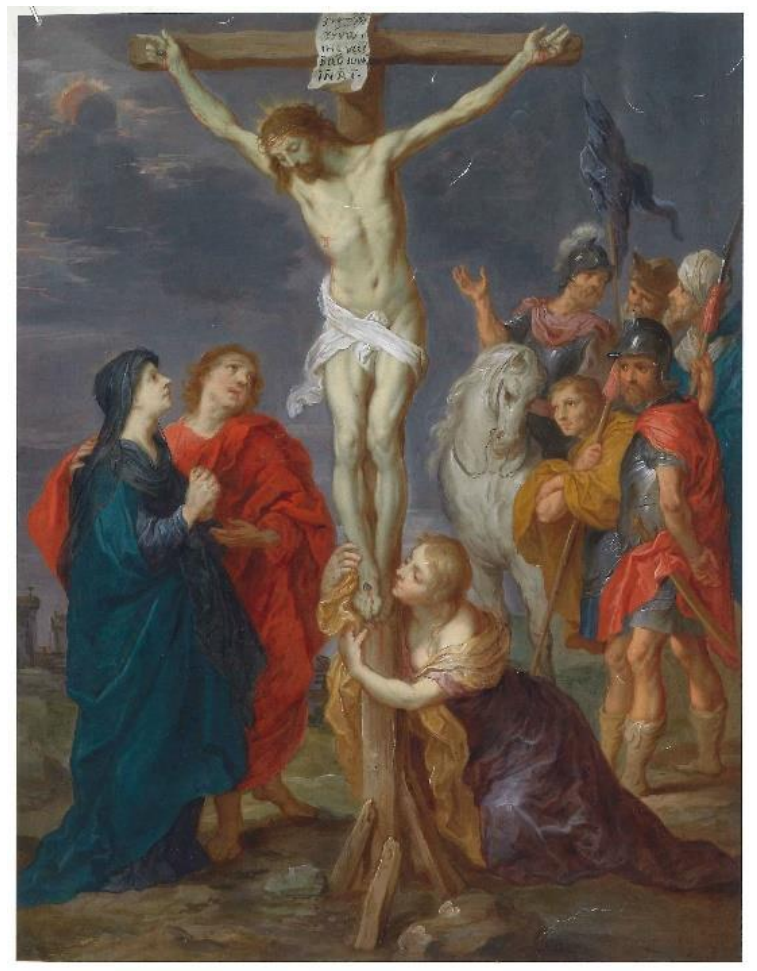

Fig. 2. Victor Wolfvoet II, Calvario, colección privada, ( ) Foto autor

atribuido en el mercado artístico al taller de Rubens ${ }^{11}$. (Fig. 2) Esta Crucifixión la repite el pintor sobre tabla en la versión de la antigua colección Gerstenmaier $(64 \times 43,5 \mathrm{~cm})^{12}$; (Fig. 3a) así como en otra réplica que estuvo considerada original de Abraham van Diepenbeeck (T., $58 \times 43,5 \mathrm{~cm})^{13}$. (Fig. 3b)

La comparación entre las tres pinturas es suficiente para advertir las pequeñas diferencias entre ellas. Está más a la vista el torso con coraza del centurión a caballo en la versión en cobre respecto a las otras réplicas. El Crucificado queda ligeramente más alejado del resto de figuras, la claridad del fondo azul es mayor, al igual que la riqueza del vestido de la Magdalena, y la delicadeza en el tratamiento de los rostros. El ligero desplazamiento del grupo a la derecha contribuye a fijar mejor la atención en los protagonistas del drama. Las diferencias están lejos de alcanzar el mimetismo al boceto de Rubens conservado en la Rockoxhuis de Amberes (inv. n.0 77.124; T. 51 x $38,5 \mathrm{~cm}$.). (Fig. 4)

Es este boceto el que sirvió a Victor Wolfvoet de referente para el cobre y las dos tablas antes citadas. Un boceto previsto por Peter Paul Rubens para

\footnotetext{
${ }^{11}$ Colonia, Lempertz (17 de noviembre, 2012), no 1133.

12 Como anónimo seguidor de Rubens en el catálogo de 2007. Pintura Flamenca Colección Gerstenmaier, cat. exp., 21 de mayo-5 de agosto, (Granada: CajaGranada, 2007), p. 40. Díaz Padrón, "Un Calvario", pp. 82-84.

13 París, Tajan (26 de junio, 2008, n. 0 41). Díaz Padrón, "Una segunda réplica", pp. 74-75.
} 


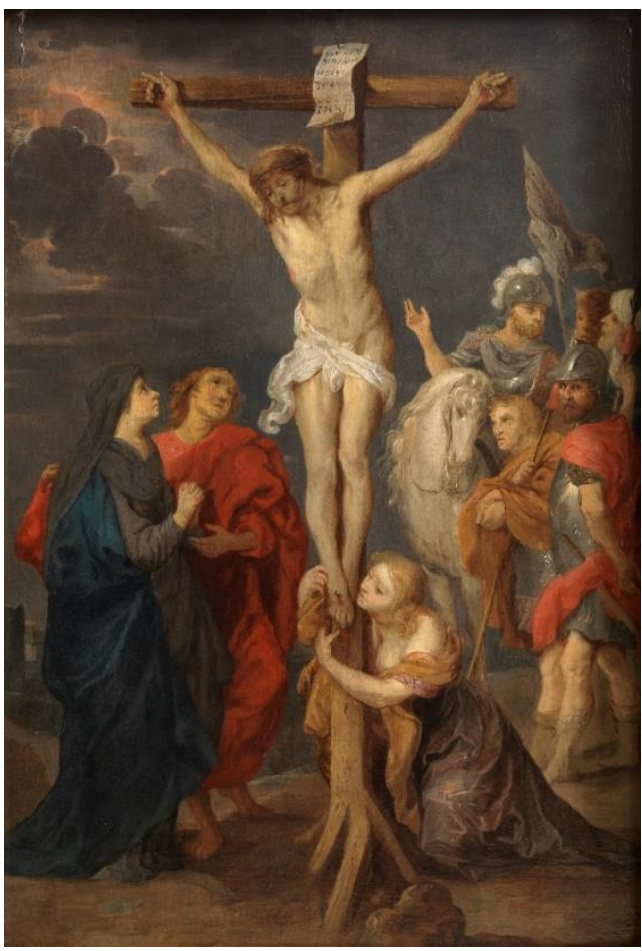

Fig. 3a. Victor Wolfvoet II, Calvario. Madrid, antigua colección Gerstenmaier ()

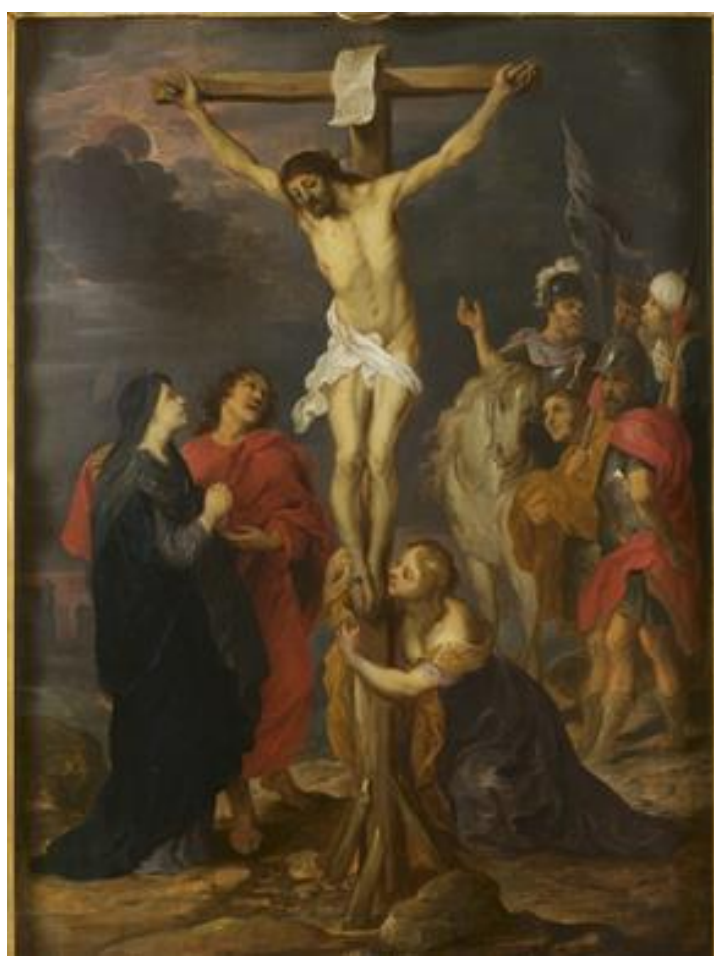

Fig. 3b. Victor Wolfvoet II, Calvario. Colección privada, ( ) Foto autor

la Crucifixión con destino la iglesia de san Miguel de Gante que no se llegó a realizar. Por lo que es evidente que Victor Wolfvoet tuvo que partir de este boceto, ignorado en estudios posteriores. Fue reconocido por primera vez en el catálogo de la exposición de Zúrich de $1955^{14}$, y con extensa bibliografía en la de Colonia de $1968^{15}$.

Este boceto de Rubens debió gozar de gran prestigio, a juzgar por el esmerado estudio que le dedica Richard Judson en el volumen del Corpus Rubenianum dedicado a las escenas de la pasión ${ }^{16}$. Fue un encargo para la capilla de Santa Úrsula de la iglesia de san Miguel de Gante. ${ }^{17}$ Rubens no pudo llevar el proyecto a gran formato al tener que viajar a Madrid en 1628, con una misión política bien conocida ${ }^{18}$. Esto motivó que el encargo recayera en Anton Van Dyck, que en gran medida siguió la idea del maestro ${ }^{19}$. Aunque Rubens no llegara a realizar el lienzo en gran formato para la capilla de san

${ }^{14}$ Alte Meister aus der Sammlung E. Bührle, cat. exp., (Zürich: Schloss Jegenstorf, 1955) no 20.

15 Weltkunst aus Privatbesitz ind Dr. Kunsthalle Köln Ausstellung det Kölner Museen, cat. exp., (Köln: Kölner Museen, 1968), nº F33.

${ }_{16}$ J. Richard Judson, The Passion of Christ, Corpus Rubenianum, Ludwig Burchard, part VI, (TurnhoutLondon: Harvey Miller Publishers, 2000), p. 133, no 34, y Fig. 104.

$17 \mathrm{Ph}$. Kervyn de Volkaersbeke publica en parte los archivos de San Miguel que hablan del encargo a Rubens en 1627 por 800 guilders estipulándose que mediría XIIII pies de altura (aprox. $417 \mathrm{~cm}$.). Ph. Kervyn de Volkaersbeke, Les Eglises de Gand, II, (Gand: Hebbelynck, 1858), pp. 92-93, nota 1, cit. J. Richard Judson, Passion of Christ, pp. 134-135.

18 Simon A. Vosters, Rubens y España. Estudio artístico-literario sobre la estética del Barroco, (Madrid: Cátedra, 1990), p. 116; Alejandro Vergara, The Presence of Rubens in Spain, (New York: University press, 1994), pp. 33-34.

${ }^{19}$ (Lienzo, 400 x $292 \mathrm{~cm}$.) Susan Barnes, Nora de Poorter, Oliver Millar, Horst Vey, Van Dyck: A Complete Catalogue of the Paintings, (New Haven-London: Paul Mellon Centre for Studies in British Art. Yale University Press, 2004), p. 262, no III.24. 


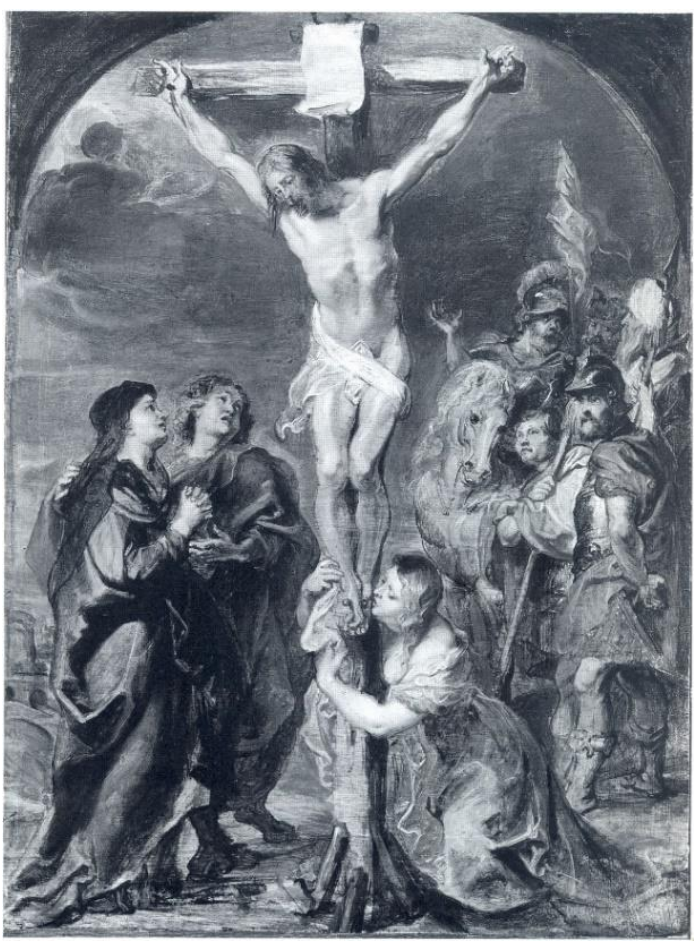

Fig. 4. Peter Paul Rubens, Calvario. Boceto, Amberes, (C) Rockoxhuis

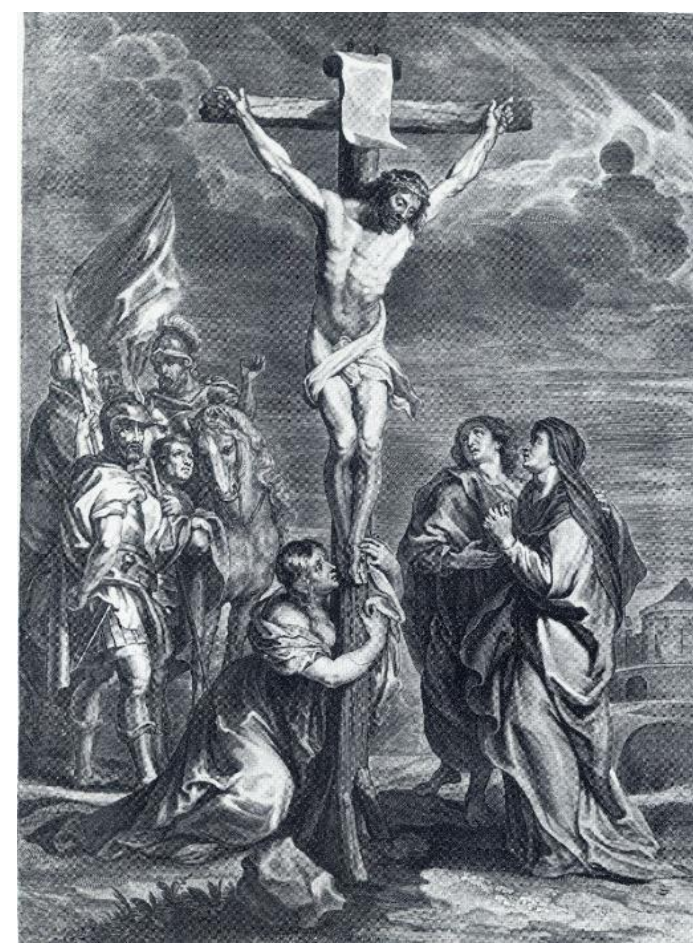

Fig. 5. Jacob Neefs siguiendo a Peter Paul Rubens, Calvario. Grabado. Amsterdam, Rijksmuseum (RP-OB-67.967)

Miguel, no fue impedimento para la influencia y notoriedad que alcanzó la composición, hecho que prueba su divulgación en los grabados de Jacob Neefs y de Jan Baptist Barbé20. (Fig. 5) Victor Wolfvoet añade en sus Calvarios la inscripción con la burla "rey de los judíos" en la cartela sobre la cruz, que Rubens dejó en blanco en el boceto.

Peter Paul Rubens concibió la composición de medio punto, y Victor Wolfvoet la adaptó al formato rectangular habitual para la pintura de gabinete. El sentimiento de la obra sigue la descripción de los misales de san Francisco ${ }^{21}$. La Virgen reprime el Ilanto con la luz bañando a Cristo y a la Magdalena. La oscuridad envolvente del celaje contribuye al clima dramático con las nubes ocultando la luna. Este fondo sombrío contrasta con los rojos y verdes esmeraldas de las vestiduras de los asistentes, unos llorando y otros indiferentes. Advertimos que la copia de Victor Wolfvoet consigue captar el pathos emocional de Rubens. Igual que el maestro, omite a los ladrones y los nimbos de las figuras divinas, para poner en valor la carga humana del trágico momento. La composición fue muy repetida. Además de los modelos conocí-

20 Carl Gottfried Voorhelm Schneevoogt, Catalogue des estampes gravées d'après P.P. Rubens avec I'indication des collections où se trouvent les tableaux et les gravures, (Haarlem: Les Héritiers Loosjes, $1873)$, p. 47, no 324 y no 325.

${ }^{21}$ John B. Knipping, Iconography of the Counter Reformation in the Netherlands, Leiden, II, (NieuwkoopLeiden: A. W. Sijthoff, 1974), p. 246, nota 13. 


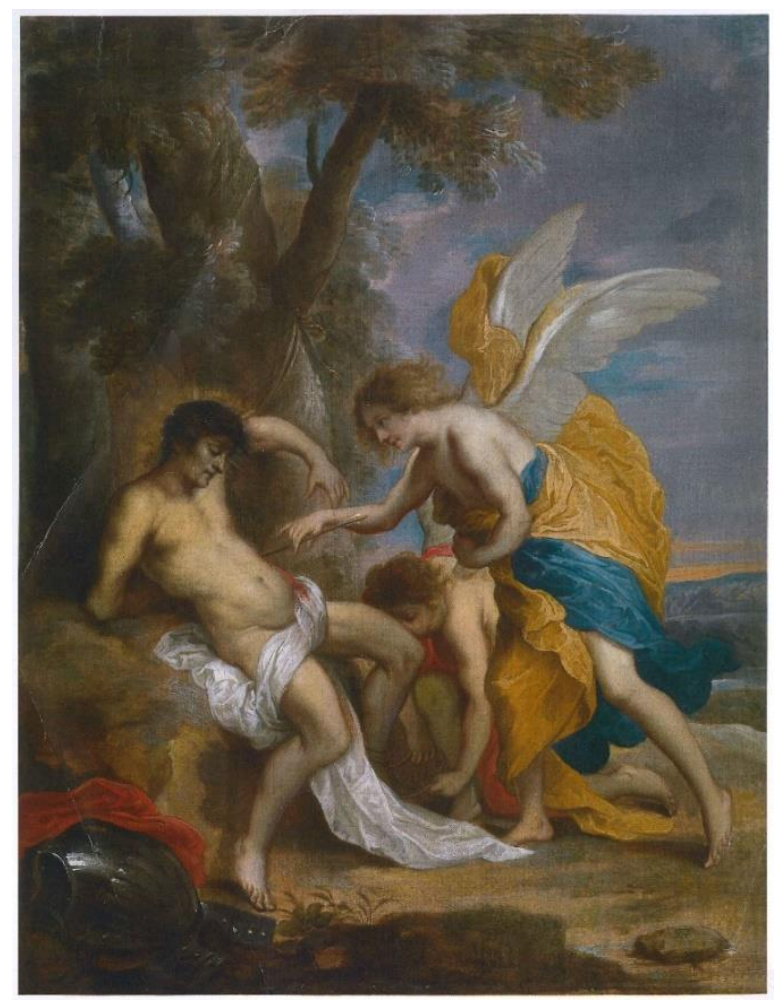

Fig. 6 Victor Wolfvoet, San Sebastián asistido por los ángeles. Colección privada, @ Foto autor

dos, por documentación sabemos que Victor Wolfvoet legó a su tía, Livine van Geemont, una versión del mismo tema ${ }^{22}$.

La estrecha dependencia de Victor Wolfvoet de composiciones no sólo del maestro sino también de Anton van Dyck, la advertimos en el Martirio de san Sebastián, catalogado en su momento como Peter Thijs (Amberes, 16241677) ${ }^{23}$. (Fig. 6) Realizado sobre tabla, asume el estilo y personalidad del original de Van Dyck conservado en el Ermitage de San Petersburgo (inv. n.o 608) ${ }^{24}$. (Fig. 7)

La pintura es de calidad, pero lejos del estilo de Peter Thijs ${ }^{25}$, donde la precisión y fino modelado de este pintor se aleja de la factura más suelta y vibrante que delata el trabajo de Victor Wolfvoet. El diseño de la nariz, los modelos y la técnica inciden en esta misma propuesta.

\footnotetext{
${ }^{22}$ F. Jos van den Branden, Geschiedenis der Antwerpsche Schilderschool, 2 vol., (Antwerpen: Buschmann, 1883), p. 799.

23 (Tabla, 41,2 x $32 \mathrm{~cm}$.) Atribuida a Peter Thijs por Alex Heindrich y Alexander Strasoldo. Viena, Dorotheum, (17 de abril, 2013, n.0 792).

${ }^{24}$ (Tabla, $147 \times 108$ cm.) Destruido en la Segunda Guerra Mundial. Matías Díaz Padrón, "Van Dyck: San Sebastián asistido por un ángel del marqués de la Ensenada identificado en el Hermitage de San Petersburgo", Boletín del Museo de Instituto Camón Aznar, no 110, (2012), pp. 127-142; M. Díaz Padrón, Jahel Sanzsalazar, colab. y Ana Diéguez-Rodríguez, colab., Van Dyck en España, vol. 1., (Barcelona: Editorial Prensa Ibérica, 2012), pp. 400-403, cat. n.o 41.

25 Danielle Maufort, De Antwerpse Kunstschilder Peeter Thijs de Oude (1624-1677), Een enadering aan de hand van zjn historiestukken, Verhandeling van licenciaat, prom. H. Vlieghe, (Leuven: Katholieke Universiteit, 1986).
} 


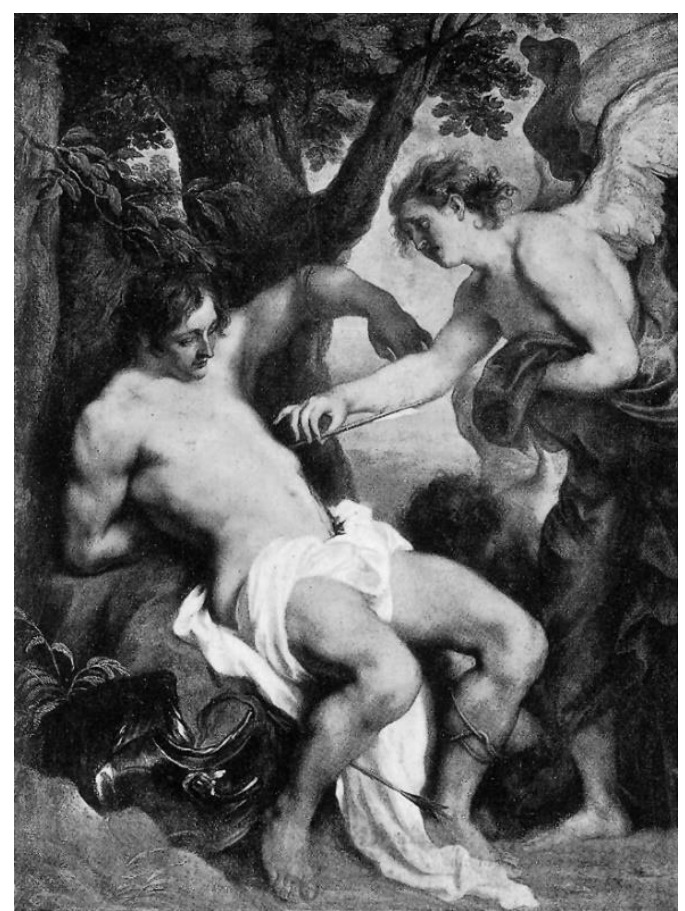

Fig. 7. Anton Van Dyck, San Sebastián asistido por los ángeles. San Petersburgo, Museo del Hermitage de San Petersburgo (destruido en la Segunda Guerra Mundial)

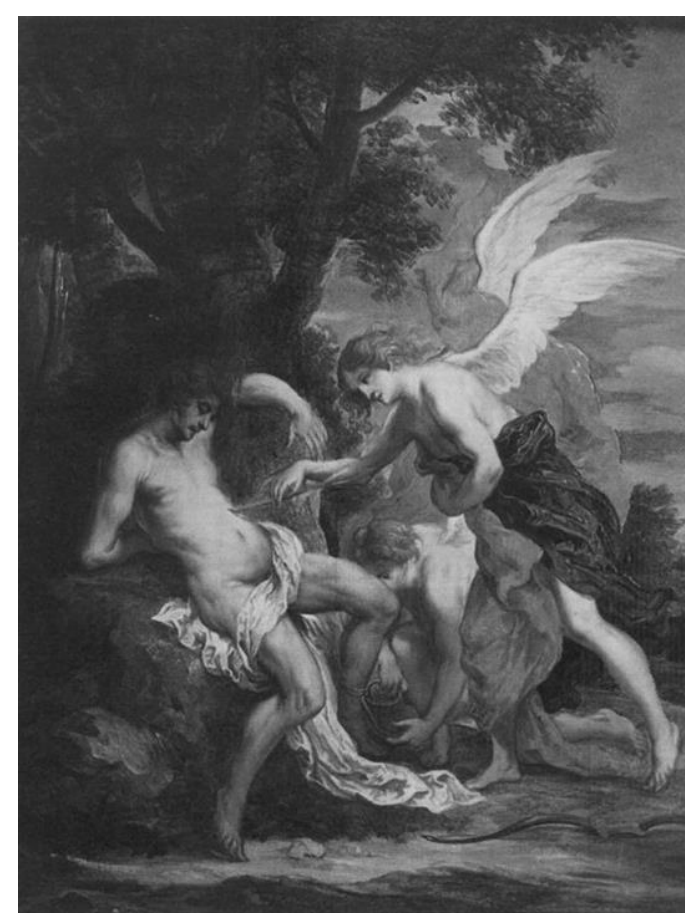

Fig. 8. Victor Wolfvoet II, San Sebastián asistido por los ángeles. Colección C. Leegenhoec, @Foto autor

Como en el original de gran tamaño de Anton Van Dyck del Ermitage (inv. n. ${ }^{\circ}$ 608), Wolfvoet sigue la escena con extrema fidelidad. El santo está apoyado en una roca y junto a un árbol robusto donde fue blanco de las flechas. El brazo izquierdo pende de las ataduras y el derecho está atado a su espalda, en una postura que delata la entrega a su destino. El trágico momento no limita la belleza y la estética del sentimiento. El modelo apolíneo del santo no está acorde con la clientela española ${ }^{26}$. El ángel extrae la flecha ocupando el lugar de santa Irene. Una visión acorde con las consignas de la contrarreforma27, y fiel a los escritos de Santiago de la Vorágine: "Sebastián, pese a la gravedad del tormento a que fue sometido, no llegó a fallecer; después que los soldados se ausentaron, alguien lo desató del árbol y lo liberó"28. Es interesante recordar que, para la postura del santo, Van Dyck se sirve del Fauno Barberini de la gliptoteca de Múnich, que está en la colección del cardenal Francesco Barberini al menos desde $1628^{29}$. El original de Van

\footnotetext{
${ }^{26}$ Louis Reáu, Iconographie de l'art chrétien, III/3, (Paris: Presses Universitaires de France, 1958), p. 1193.

${ }^{27}$ Reáu, Iconographie, III/3, p. 1198; Knipping, Iconography, pp. 128-136.

28 Santiago de la Vorágine, La leyenda dorada, (trad. del latín Fray José Manuel Macías), (Madrid: Alianza Editorial: 1982), p. 115.

${ }^{29}$ (Mármol, $215 \mathrm{~cm}$. de alto). Se dice que fue descubierta en los alrededores del Castello di Sant 'Angelo. Fue reproducida en un grabado publicado en 1642, y restaurada sucesivamente. Comparada por Cassiano del Pozzo con el Torso Belvedere. Francis Haskell y Nicolas Penny, Taste and the Antique. The Lure of Classical Sculpture, 1500-1900, (New Haven-Londres: Yale University Press, 1981), p. 202, no 33.
} 


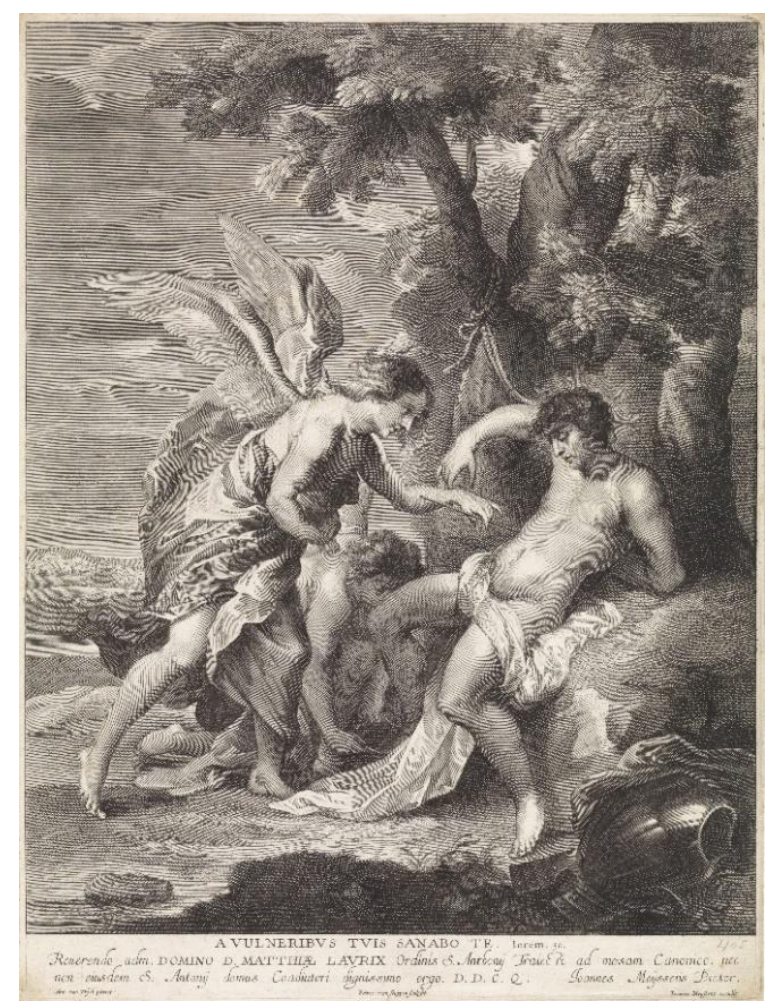

Fig. 9. Pieter van Schuppen siguiendo a Anton Van Dyck, San Sebastián asistido por los ángeles. Grabado, Amsterdam, Rijksmuseum (RP-P-OB-77.679)

Dyck estuvo en España, de donde pasó a Catalina la Grande de Rusia y al Ermitage, quizá regalo de Carlos III y Carlos IV ${ }^{30}$.

Uno al estudio de esta tabla, otra copia con variantes de igual calidad en la colección C. Leegenhoeck ${ }^{31}$, que Hairs atribuyó a Willeboirts Bosschaert y considero también de Victor Wolfvoet, siguiendo el grabado de Pieter van Schuppen ${ }^{32}$. (Figs. 8 y 9)

Completando esa dependencia evidente de Wolfvoet de los modelos de los maestros, está el cobre de Santa Cecilia tocando el clavicordio de colección privada bilbaína $(50 \times 38 \mathrm{~cm} \text {. })^{33}$ (Fig. 10). Repite en sentido invertido el ori-

\footnotetext{
${ }^{30}$ Erik Larsen, The Paintings of Anton Van Dyck, II, (Freren: Luca Verlag, 1988), p. 273, n 674; Barnes et al., Van Dyck, p. 287, no III.53.

31 (Tabla, 38,2 x 29,2 cm.) Marie Lousie Hairs, Dans le sillage de Rubens. Les peintres d'Histoire anversois au XVIIème siècle, (Liège: Université de Liège, 1977), p. 242. De hecho, en la reciente monografía sobre Bosschaert no figura esta obra. Axel Heinrich, Thomas Willeboirts Bosschaert (1613-1654). Ein Flämischer Nachfolger Van Dycks, (Turnhout: Brepols Publishers, 2003).

32 Con la inscripción: A VULNERIBUS TVIS SANABO TE. Ierm, 30. / Reverendo adm. DOMINO D.MATTHIAE LAVRIX Ordini S. Anthony Traecti ad mosam CAnonico, nec / non eiusdem S. Anthony domus Coadiutori dignísimo ergo. D.D.C.Q. Iones Meÿssens Pictor. / (abajo a la izda.): Ant. Van Dÿck pinxit (en el centro): Petrus van Scuppen sculpsit (a la derecha): Iones Meyssens excudit Amsterdam. The New Hollstein. Dutch \& Flemish Etchings, Engravings and Woodcuts 1450-1700. Anthony van Dyck, Simon Turner, comp. y Carl Depauw, ed., Vol. VIII, (Rotterdam: Sound \& Vision, 2002), p. 129, no 614.

${ }^{33}$ Galería Astarloa de Bilbao, como obra de Willem van Herp. Años antes aparece en Barcelona con la misma atribución. Barcelona, Balclis (17 de octubre de 2013, no 1456).
} 


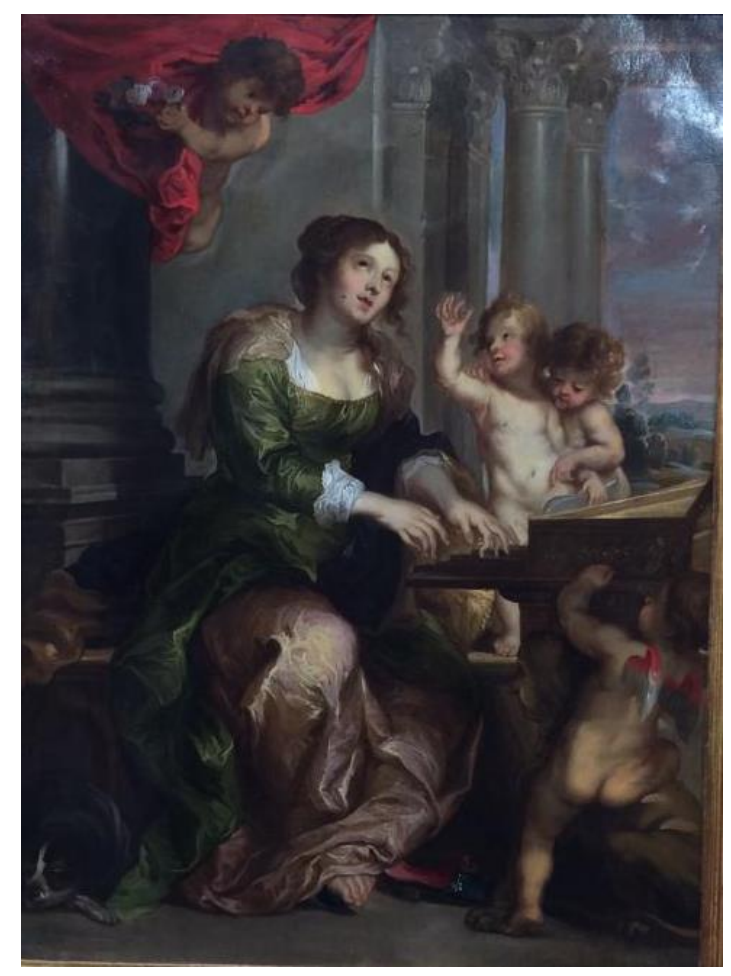

Fig. 10. Victor Wolfvoet, Santa Cecilia tocando el clavicordio. Bilbao, colección privada, ( ) Foto autor

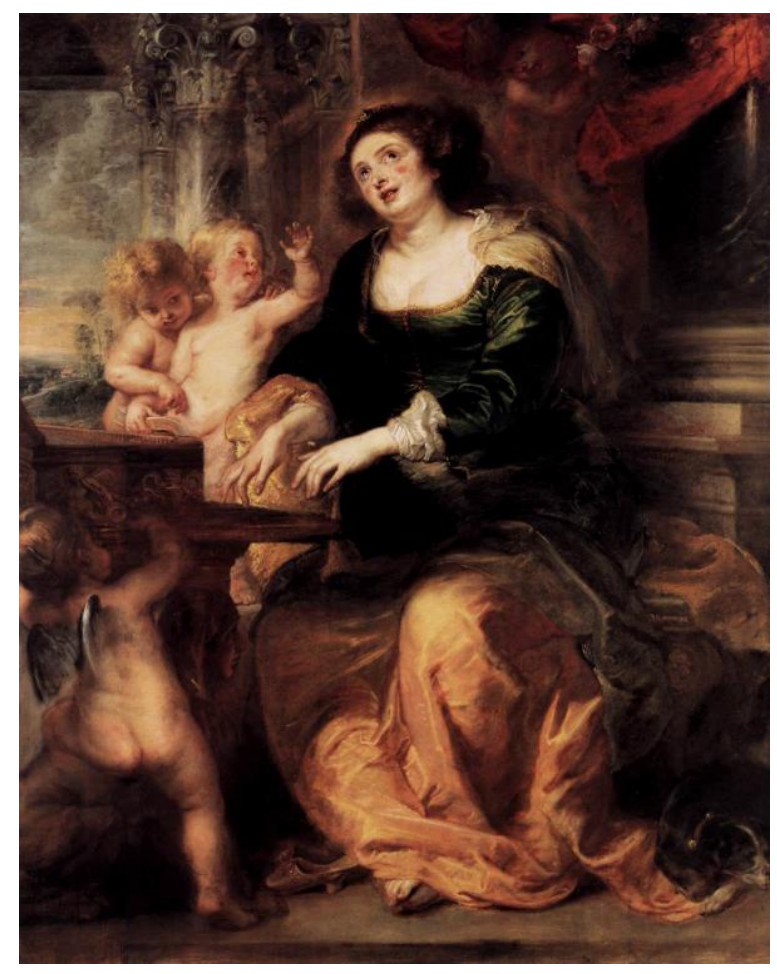

Fig. 11. Peter Paul Rubens, Santa Cecilia tocando el clavicordio. Berlín, () Gemäldegalerie der Staatlichen Museen zu Berlin (inv. $\mathrm{n}^{\mathbf{0}}$ 781)

ginal de Peter Paul Rubens de la Gemäldegalerie de Berlín (inv. n. 0 781) , $^{34}$ donde el rostro de la santa recuerda los rasgos de la segunda esposa del pintor, Helena Fourment (Fig. 11). Escapando a los límites de este estudio, nos interesa recordar el posible vínculo de esta pintura con Jacob van Ophem, importante cortesano de Bruselas, señor de Neden y Over Hembeek ${ }^{35}$, en agradecimiento de los herederos de Rubens por garantizar el pago de pinturas encargadas por el rey de España ${ }^{36}$.

Este cobre estuvo atribuido a Willem van Herp (Amberes, ca. 1613-1672), pintor de personalidad bien definida y también seguidor de Peter Paul Rubens en algunas ocasiones, pero sin el servilismo que se aprecia en Victor Wolfvoet.

34 (Tabla, 180,6 x 142,7 cm.) Gemäldegalerie der Staatlichen Museen zu Berlin (inv. no 781). Rüdiger Klessmann "Rubens's Saint Cecilia in the Berlin Gallery after cleaning", The Burlington Magazine, vol. 107, no 752, July, (1965) pp. 550-559.

35 Relacionado con la corte de los archiduques Isabel y Alberto, su palacio de Bruselas debió contar con una importante colección artística, a juzgar por las obras que se conocen de su colección. Susan Koslow, The Noble Estate. Seventeenth-Century Still-Life and Animal Painting in the Spanish Netherlands, (Antwerpe: Fons Mercator Paribas, 1995), pp. 109-142. Agradezco a Ana Diéguez Rodríguez esta información.

36 "Jacques van Ophem, Ridder, Raedt ende Rentmeester Generaal vande Domeynen van Syne Majesteyt int quartier van Brussel": Pierre Génard, "De Nalatenschap van P. P. Rubens / La succession de P. P. Rubens'", Antwerpsch Archievenblad / Bulletin des Archives d'Anvers, II, (1865-1866), p. 85. Pierre Génard, P. P. Rubens, Aanteekeningen over den grooten Meester en zijne Bloedverwanten, (Antwerpen: Kockx), 1877, pp. 44-45; Max Rooses, L'Oeuvre de Peter Paul Rubens. Histoire et Description de ses Tableaux et Dessins, II, (Anvers: J. Maes, 1888), p. 240; Klessmann, "Rubens's Saint Cecilia", p. 550, nota 1; Hans Vlieghe, Saints, I, Corpus Rubenianum Ludwig Burchard, VIII, (Brussels: Arcade Press, 1972), pp. 128-129; Jeffrey M. Muller, Rubens: The Artist as a Collector, (Oxford: Princeton University Press, 1989), pp. 113-114, no 93. Agradezco a Magdala García Sánchez de la Barreda todas las precisiones sobre este asunto. 


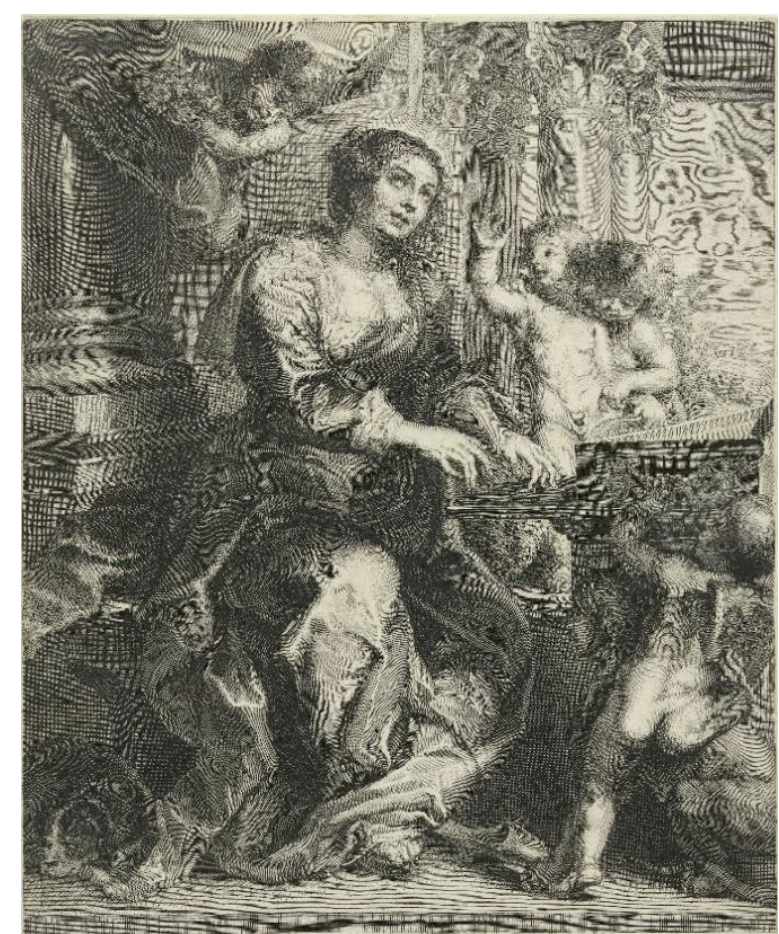

Fig. 12. Schelte à Bolswert siguiendo a Peter Paul Rubens, Santa Cecilia tocando el clavicordio. Grabado, Amsterdam, Rijksmuseum (RP-P-BI-2546)

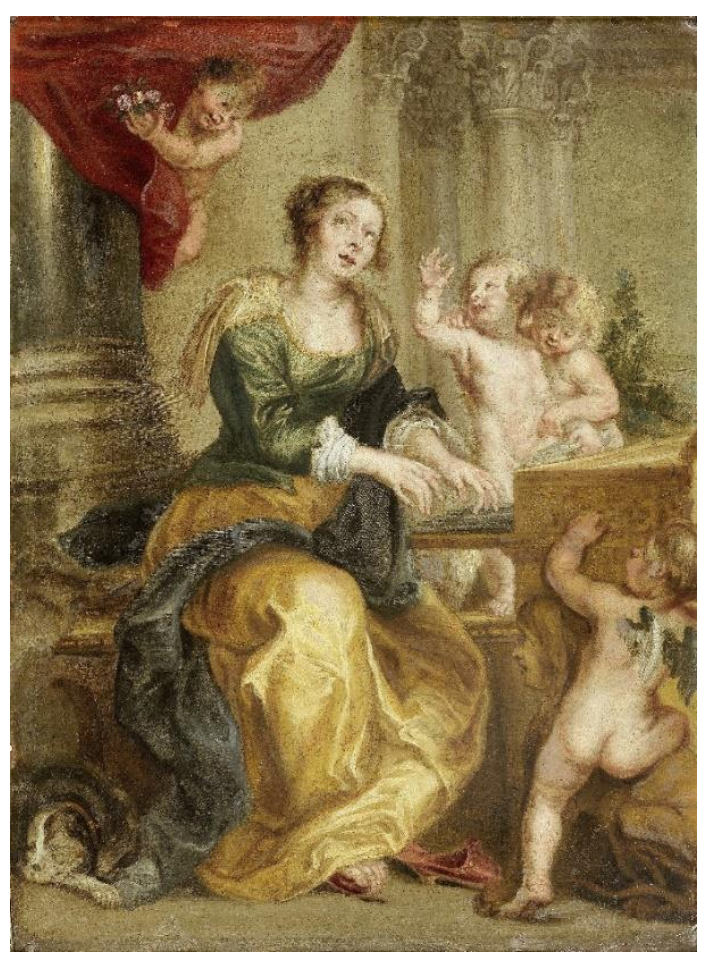

Fig. 13. Simon de Vos, Santa Cecilia tocando el clavicordio. Colección privada, () Foto autor

De hecho, esta pintura está lejos de las figuras estilizadas, propias de Van Herp, y coincide en estilo con las maneras de Wolfvoet. Posiblemente el pintor se valiera del grabado de Hans Witdoeck y de Schelte à Bolswert siguiendo a Rubens $^{37}$. (Fig. 12) Todo es idéntico, salvo el muro y la galería de columnas del fondo abiertas al paisaje. La diferencia está en la juventud y expresión más afín con el grabado, invirtiendo la colocación y acentuando la altura de las columnas y techo de la estancia. Todo da idea de estar ante una de las pinturas más cuidadas del artista. Una pintura exquisita y típica de Victor Wolfvoet.

La santa esta frente al órgano cantando con la mirada en éxtasis hacia lo alto. Es interesante destacar la belleza del vestido con ricos reflejos de la luz del fondo, y cómo los angelitos participan de los acordes de la música y el aroma de las rosas.

El modelo debió ser muy imitado, pues aparte de Wolfvoet, constatamos una repetición de Simon de Vos, también en cobre (36'5 × $28^{\prime} 4 \mathrm{~cm}$ ), procedente de la antigua colección del marqués de Blaisel (1790-1870) ${ }^{38}$. (Fig. 13)

37 Tercer estadio con la inscripción: FICTA PRIOR POST HAC SILEAT MIRACLA VETUSTAS; NUNC ORBI MAIOR SVRREXIT CAECILIS ORPHEO / ORPHEUVS ET SILEAT SAXA TRAHENTE LYRA. ILLE FERAS; CAELVUM HACE AD SVA PLECTRA TRAHIT / (abajo a la derecha): P.P. Rubens pinxit / S. à Bolswert / (abajo en el centro): Cum gratia et privilegio Regis J de berti. / (abajo a la derecha): Gillis Hendricx excudit. Hollstein, Dutch, vol. 53, no 20-II.

${ }^{38}$ Londres, Bonhams (23 de octubre, 2019), no 102. Vlieghe, Saints, I, p. 128, en no 82 (copia 1). 
En fin, los estudios dedicados a Victor Wolfvoet van desvelando una sorprendente actividad que da idea de la notoriedad de este pintor trabajando en la primera mitad del siglo XVII, pero que ha estado olvidado por la crítica hasta hace poco tiempo. Es, entre los numerosos maestros de calidad que siguen los modelos de Rubens, el que alcanza mayor fidelidad y ferviente entrega a las propuestas del maestro. 
Bibliografía:

Barnes et al. 2004: Susan Barnes, Nora de Poorter, Oliver Millar, Horst Vey, Van Dyck: A Complete Catalogue of the Paintings, (New Haven-London: Paul Mellon Centre for Studies in British Art. Yale University Press, 2004).

Díaz Padrón 1999: Matías Díaz Padrón, "Dos cobres de Victor Wolfvoet en el Museo San Carlos de Méjico", Boletín del Seminario de Arte y Arqueología: $B S A A$, n. 0 65, (1999), pp. 323-328.

Díaz Padrón 2006: Matías Díaz Padrón, "Tres cobres restituidos a Victor Wolfvoet, el más fiel seguidor de Rubens" Archivo Español de Arte, LXXIX, n. 0 316, (2006), pp. 403-411.

Díaz Padrón 2008: Matías Díaz Padrón, "Una adoración de los reyes de Víctor Wolfvoet en el coleccionismo madrileño", Tendencias del Mercado del Arte, n. ${ }^{\circ}$ 18, (2008), pp. 32-33.

Díaz Padrón 2012: Matías Díaz Padrón, "Tres nuevos cobres de Víctor Wolfvoet con la guerra y la paz bajo la consigna de Rubens", Archivo Español de Arte, LXXXV, 337, enero-marzo, (2012), pp. 75-94.

Díaz Padrón 2012: Matías Díaz Padrón, "Una Andrómeda con Perseo y Pegaso en la lejanía de Victor Wolfvoet atribuida a David Teniers en la galería Christie's de Nueva York", Tendencias del mercado del arte, no 53, mayo (2012), pp. 92-94.

Díaz Padrón 2012: Matías Díaz Padrón, "Van Dyck: San Sebastián asistido por un ángel del marqués de la Ensenada identificado en el Hermitage de San Petersburgo", Boletín del Instituto y Museo Camón Aznar, no 110, (2012), pp. 127-142.

Díaz Padrón 2012: Matías Díaz Padrón, Jahel Sanzsalazar, colab. y Ana Diéguez-Rodríguez, colab., Van Dyck en España, (Barcelona: Editorial Prensa Ibérica, 2012).

Díaz Padrón 2013: Matías Díaz Padrón, "Un Calvario de Victor Wolfvoet en la colección Gerstenmaier", Tendencias del mercado del arte, no 68, (2013), pp. 82-84.

Díaz Padrón 2017: Matías Díaz Padrón, "La Visitación de Saint- Jacques de Amberes ¿Victor Wolfvoet o Simon de Vos?", Philostrato. Revista de Historia y Arte, n. 0 1, (2017), pp. 43-55, (DOI: https://doi.org/10.25293/philostrato.2017.03)

Díaz Padrón 2018: Matías Díaz Padrón, "Algo más sobre Victor Wolfvoet. Una segunda réplica de la Crucifixión atribuida a Abraham van Diepenbeeck", Philostrato. Revista de historia y arte, no extraordinario 1, (2018), pp. 296304, (DOI: https://doi.org/10.25293/philostrato.2018.11) 
Díaz Padrón 2020: Matías Díaz Padrón, "Una segunda réplica de la Crucifixión de Victor Wolfvoet atribuida a Abraham Diepenbeeck", Tendencias del mercado del arte, n. ${ }^{\circ} 136$, noviembre, (2020), pp. 74-75.

Duverger 1992: E. Duverger, Antwerpse Kunstinventarissen uit de Zeventiende Eeuw, vol. 6, Fontes Historiae Artis Neerlandicae, (Brussel: Paleis der Academiën, 1992).

García Cueto 2021: David García Cueto, "The Reflection of 'Flandes' in the Spanish Royal Collections after Rubens in the Patrimonio Nacional", en Copies of Flemish Masters in the Hispanic World (1500-1700). Flandes by Substitution, eds. Eduardo Lamas y David García Cueto, (Turnhout: Brepols, 2021), p. 199

Génard 1865-1866: Pierre Génard, "De Nalatenschap van P. P. Rubens/ La succession de P. P. Rubens", Antwerpsch Archievenblad/ Bulletin des Archives d'Anvers, II, (1865-1866), pp. 69-179.

Génard 1877: Pierre Génard, P. P. Rubens, Aanteekeningen over den grooten Meester en zijne Bloedverwanten, (Antwerpen: Kockx, 1877).

Granada 2007: Pintura Flamenca Colección Gerstenmaier, cat. exp., (21VI/5-VIII-2007), (Granada: CajaGranada, 2007).

Hairs 1977: Marie Lousie Hairs, Dans le sillage de Rubens. Les peintres d'Histoire anversois au XVIIème siècle, (Liège: Université de Liège, 1977).

Haskell y Penny 1981: Francis Haskell y Nicolas Penny, Taste and the Antique. The Lure of Classical Sculpture, 1500-1900, (New Haven-Londres: Yale University Press, 1981).

Heinrich 2003: Axel Heinrich, Thomas Willeboirts Bosschaert (1613-1654). Ein Flämischer Nachfolger Van Dycks, (Turnhout: Brepols Publishers, 2003).

Hollstein Dutch: Frederick de Wit to Lieven de Witte, Christiaan Schuckman, Ilja M. Veldman, Jeroen de Scheemaker comp., D. de Hoop Scheffer ed., Dutch \& flemish etchings, engravings and woodcuts ca. 1450-1700, vol. 53, (Rotterdam: Sound \& Vision Publishers, 1999).

Judson 2000: J. Richard Judson, The Passion of Christ, Corpus Rubenianum, Ludwig Burchard, part VI, (Turnhout-London: Harvey Miller Publishers, 2000).

Kervyn de Volkaersbeke 1858: Philippe Augustin Chrétien Kervyn de Volkaersbeke, Les églises de Gand, II: Eglises paroissiales et oratoires, (Gand: Hebbelynck, 1858).

Klessmann 1965: Rüdiger Klessmann "Rubens's Saint Cecilia in the Berlin Gallery after cleaning", The Burlington Magazine, vol. 107, n. ${ }^{\circ}$ 752, July, (1965), pp. 550-559.

Knipping 1974: John B. Knipping, Iconography of the Counter Reformation in the Netherlands, 2 vols., (Nieuwkoop-Leiden: A. W. Sijthoff, 1974). 
Köln 1968: Weltkunst aus Privatbesitz ind Dr. Kunsthalle Köln Ausstellung det Kölner Museen, cat. exp. (18-V- 4-VIII-1968), (Köln: Verlag, 1968).

Koslow 1995: Susan Koslow, The Noble Estate. Seventeenth-Century StillLife and Animal Painting in the Spanish Netherlands, (Antwerpe: Fons Mercator Paribas, 1995).

Lacarra Ducay y Lozano López 2015: M. Carmen Lacarra Ducay y Juan C. Lozano López, coords., Aragón y Flandes. Un encuentro artístico. Siglos XVXVII, (Zaragoza: Universidad, 2015).

Larsen 1988: Erik Larsen, The Paintings of Anton Van Dyck, 2 vols., (Freren: Luca Verlag, 1988).

Martin y Schepers 2016: Gregory Martin y Bert Schepers, "Two Antwerp cabinets decorated by Victor Wolfvoet II", The Burlington Magazine, 158, no 1363, (2016), pp. 793-802.

Maufort 1986: Danielle Maufort, De Antwerpse Kunstschilder Peeter Thijs de Oude (1624-1677), Een enadering aan de hand van zjn historiestukken, Verhandeling van licenciaat, prom. H. Vlieghe, (Leuven: Katholieke Universiteit, 1986).

Muller 1989: Jeffrey M. Rubens: The Artist as a Collector, (Oxford: Princeton University Press, 1989).

New Hollstein: The New Hollstein. Dutch \& Flemish Etchings, Engravings and Woodcuts 1450-1700. Anthony van Dyck, Simon Turner, comp. y Carl Depauw, ed., Vol. VIII, (Rotterdam: Sound \& Vision, 2002).

Reáu 1958: Louis Reáu, Iconographie de I'art chrétien, III/ 3, (Paris: Presses Universitaires de France, 1958).

Rombouts y Van Lerius 1872-1961: Philips Rombouts y Theodor van Lerius, De Liggeren en Andere Historische Archieven, vol. 2, (s'Granvenhage, 1872/1961).

Rooses 1888: Max Rooses, L'Oeuvre de Peter Paul Rubens. Histoire et Description de ses Tableaux et Dessins, II, (Anvers: J. Maes, 1888).

Schepers 2017: Bert Schepers, "Copy/paste Rubens: the art of Victor II Wolvoet. Newly identified works in the Spanish Royal Collections", en Las copias de obras maestras de la pintura en las colecciones de los Austrias y el Museo del Prado. Actas del congreso internacional. Museo del Prado, junio, 2017, (Madrid: Museo Nacional del Prado, 2021), pp. 106-115.

Van den Branden 1883: Frans Jozef Peter van den Branden, Geschiedenis der Antwerpsche Schilderschool, 2 vols., (Antwerpen: Buschmann, 1883).

Vergara 1994: Alejandro Vergara, The Presence of Rubens in Spain, (New York: University press, 1994).

Vlieghe 1972: Hans Vlieghe, Saints, I, Corpus Rubenianum Ludwig Burchard, part. VIII, 2 vols., (Brussels: Arcade Press, 1972). 
Voorhelm Schneevoogt 1873: Carl Gottfried Voorhelm Schneevoogt, Catalogue des estampes gravées $d$ 'après P.P. Rubens avec I'indication des collections où se trouvent les tableaux et les gravures, (Haarlem: Les Héritiers Loosjes, 1873).

Vorágine 1982: Santiago de la Vorágine, La leyenda dorada, Fray José Manuel Macías, trad., (Madrid: Alianza Editorial, 1982).

Vosters 1990: Simon A. Vosters, Rubens y España. Estudio artístico-literario sobre la estética del Barroco, (Madrid: Cátedra, 1990).

Zürich 1955: Alte Meister aus der Sammlung E. Bührle, cat. exp., Schloss Jegenstorf. Sammlung E. Buhrle, Zürich, 21-V/31-VIII-1955, (Fraubrunnen: Glauser, 1955).

Recibido: $14 / 10 / 2021$

Aceptado: $27 / 10 / 2021$ 